\title{
基于蒙特卡罗树搜索的树图布局
}

\author{
刘婷婷, 汪云海*, 屠长河, 蒋鹏 \\ (山东大学计算机科学与技术学院 青岛 266237) \\ (cloudseawang@gmail.com)
}

\begin{abstract}
摘 要: 树图是层次数据可视化的常用方法, 已有的研究发现, 满足逐步加点原则的 3 种树图布局算法(正方化布局 算法、条状布局算法和螺旋线布局算法)在常见的 4 种优化指标(平均长宽比、稳定性、数据一致性和可读性)上各有 优缺点. 其中, 条状布局算法和螺旋线布局算法的长宽比较大, 正方化布局的长宽比仍有优化空间. 基于蒙特卡罗树 搜索，在树图布局的同时构建对应的搜索树并利用置信度上界公式作为搜索路径的选定标准，在用户可接受时间内 对 3 种布局算法的算术平均长宽比和所提加权平均长宽比分别进行了优化. 为了证明算法的有效性, 在构造出的 3 种不同分布、 7 种不同规模的单层数据集以及真实的多层数据集上测试, 并与原算法进行比较分析, 最后利用对应结 论指导用户选择更好的布局算法以及设置更恰当的参数.
\end{abstract}

关键词：层次结构数据; 树图; 蒙特卡罗树搜索; 布局算法选取

中图法分类号: TP391.41 DOI: 10.3724/SP.J.1089.2021.18682

\section{Treemap Layout Based on Monte Carlo Tree Search}

\author{
Liu Tingting, Wang Yunhai*, Tu Changhe, and Jiang Peng \\ (School of Computer Science and Technology, Shandong University, Qingdao 266237)
}

\begin{abstract}
Treemaps are a commonly used method for hierarchical data visualization. The previous research shows that three treemap layout algorithms(SQR, STR, SPI) that meet the principle of gradually adding points on four common optimization indicators(average aspect ratio, stability, consistent data, readability) have their own advantages and disadvantages. Both Strip and Spiral algorithms result in relatively large aspect ratio of layouts, and the squarified treemap has some room for optimization in the aspect ratio. Based on Monte Carlo tree search, the corresponding search tree is constructed while laying out the treemap and the upper confidence bound formula is used as the selection criterion for the search path. For the three layout algorithms, the arithmetic average aspect ratio and the newly proposed weighted aspect ratio are improved within acceptable time for users. To show the effectiveness of the algorithm, the evaluation with the constructed single-layer dataset which with 3 different distributions and 7 different scales and multi-layer real dataset is conducted and then the results are compared with the original algorithm. The guidelines for users to select a suitable layout algorithm and set proper parameters are also provided.
\end{abstract}

Key words: hierarchical data; treemap; Monte Carlo tree search; layout algorithm selection

收稿日期: 2020-09-26; 修回日期: 2020-11-18. 基金项目：国家自然科学基金(61772315, 61861136012). 刘婷婷(1996一), 女, 硕 士研究生, 主要研究方向为信息可视化; 汪云海(1984一), 男, 博士, 教授, 博士生导师, CCF 会员, 论文通讯作者, 主要研究方向为 感知驱动的信息可视化、人机交互等; 屠长河(1968一), 男, 博士, 教授, 博士生导师, CCF 会员, 主要研究方向为计算机图形学、数 字几何造型与处理、数字图形处理等; 蒋鹏(1986-), 男, 博士, 助理研究员, 主要研究方向为计算机视觉、图像处理、人工智能等. 
在大数据时代, 层次结构数据广泛出现在各 种场合之中, 由于其结构特殊性和复杂性, 人们难 以认知它的结构和分布. 层次数据可视化能够直 观地显示出层次数据的结构和分布, 帮助人们快 速地查找节点、对比节点权重的大小、发现数据中 隐藏的信息, 并更容易地挖掘出时序数据的变化 趋势等信息。

常见的层次数据可视化方法分为节点链接型 和空间填充型. 其中, 空间填充型对空间的利用率 很高, 即使在数据规模较大时也十分有效. 相比于 其他形状 ${ }^{[1-3]}$, 矩形较为规则、易于实现，可以完全 利用所给的矩形绘制区域, 空间填充型是层次数 据可视化方法中最常使用矩形.

目前常用的矩形树图布局算法有 14 种 $^{[4]}$. 评 估树图的方式有平均长宽比、数据一致性、数据稳 定性和可读性 ${ }^{[5]} 4$ 种，但现有的树图布局算法都无 法做到使 4 个指标同时达到最优. 例如，一种算法 若能得到长宽比较小的布局, 那么它的稳定性等 性质通常会很差; 反之，一种算法若能保证数据稳 定性等良好的性质, 那么它往往得不到长宽比较 小的布局. 为使层次数据可视化的结果尽可能好, 设计者应当根据不同的数据特性(如静态数据、动 态数据、数据规模大小等)和应用场景(如查找数 据、对比数据等)选择不同的布局算法.

本文根据树图布局原则对树图布局算法进行 分类，发现符合逐步加点布局原则的 3 种树图布局 算法：正方化布局算法(squarified, SQR) ${ }^{[6]}$ 、条状布 局算法 (strip，STR) ${ }^{[7]}$ 和螺旋线布局算法 (spiral, $\mathrm{SPI})^{[5]}$, 它们针对 4 种常见的评价指标都各有优劣, 比较有代表性, 其中, STR 和 SPI 布局结果的长宽 比往往不好, SQR 针对部分数据也有长宽比优化的 空间. 所以，本文主要针对符合逐步加点布局原则 的 3 种布局算法进行长宽比优化, 利用逐步加点的 布局原则结合蒙特卡罗树搜索(Monte-Carlo tree search, MCTS)设计了在可接受时间得到具有更优 长宽比布局结果的算法 (treemap based MCTS, TM-MCTS). 之后针对数据规模比较大的情况下, 常常只用先显示靠上层一点的数据, 再通过交互查 看下层的数据的现象, 本文提出加权长宽比这一概 念，并针对这一概念设计了对应的加权长宽比优化 (weighted treemap based MCTS, WTM-MCTS)算法.

为了证明算法的有效性，本文构造了 3 种不同 分布、 7 种不同规模的单层数据集以及一些多层真 实数据, 对 TM-MCTS 在不同参数设置下进行对比 分析, 针对多层数据还对比了 WTM-MCTS 布局结
果. 结果表明, TM-MCTS (WTM-MCTS) 确实能够 对对应算法的长宽比(加权长宽比)进行优化, 并且 用户可以根据不同的任务需求以及数据特性对算 法及其参数的设置进行选择.

\section{1 相关工作}

树图布局算法从提出到现在近 30 年来, 针对 不同的评价指标已有丰富的研究成果. 本节先简 单介绍树图布局算法在长宽比优化方面的相关研 究, 然后介绍 MCTS 的应用情况并讨论如何把树 图布局问题和 MCTS 相结合.

\section{1 基于长宽比优化的树图布局算法}

1992 年, Shneiderman ${ }^{[8]}$ 第 1 次提出用区域嵌套 的方式展示树结构, 并设计了横切和纵切布局算 法(slice and dice, SND). 该算法虽然很好地显示了 数据的层次结构, 但是长宽比往往很大. 为了解决 这一问题, Bruls 等 ${ }^{[6]}$ 提出的 SQR 解决了 SND 中出 现 “细长”矩形的问题, 成为到目前为止长宽比几 乎最好的树图布局算法. 但是 SQR 布局前需要做 数据排序, 导致所得布局结果的数据一致性、稳定 性和可读性都比较差. 之后的 20 多年,一直有很 多算法致力于平衡树图布局的平均长宽比、稳定 性、数据一致性和可读性 4 个指标, 如 Pivot 布局 算法 ${ }^{[9]}$, Ordered-SQR 算法 ${ }^{[10]}$, Split 布局算法 ${ }^{[11]}$, $\mathrm{STR}^{[7]}$, Hilbert 和 Moore 布局算法 ${ }^{[12]}, \mathrm{SPI}^{[5]}$ 和均匀 分块的正方化树图布局算法 ${ }^{[13]}$. 此外, Vliegen 等 ${ }^{[14]}$ 提出在不同层使用不同布局算法的混合布局算法; 张驰等 ${ }^{[15]}$ 针对 $\mathrm{SQR}$ 在数据一致性、稳定性和可读 性这 3 方面的不足，提出一种近似均分的正方化算 法. 也有很多人转而研究树图的交互 ${ }^{[16]}$ 以及节点 显示问题 ${ }^{[17]}$, 从而协助用户在大规模数据下快速 理解数据.

目前只有比较少的研究关注长宽比优化. Nagamochi 等 ${ }^{[18]}$ 提出近似最小化最大长宽比布局算法 (approximation, APP), 但与 SQR 一样, APP 需要先 做数据排序, 因此它们有着相似的缺点. $\mathrm{Lu}$ 等 ${ }^{[19]}$ 提 出一种尽量控制长宽比的树图布局算法. 刘旭 ${ }^{[20]}$ 更 进一步分析并指出了导致 SQR 性质较差的问题根 源——沿短边布局原则和贪心原则. 图 1a 中, 左 图是利用 SQR 布局的结果, 由于 SQR 沿着短边布 局, 得不到右图沿着长边布局的更优布局结果; 图 $1 \mathrm{~b}$ 中, 左图是 SQR 的布局结果, 在布局第 2 个节 点时改善了第 1 个节点的长宽比，第 1 个节点和第 2 个节点被放在了同一列，使第 3 个节点的长宽比 
很差, 而右图改善了这个情况. 刘旭 ${ }^{[20]}$ 利用深度 优先搜索优化 SQR 的长宽比, 却没有对庞大的搜 索空间进行取舍, 只是在控制搜索深度的条件下 遍历所有可能, 很难在有限的搜索次数内得到很 好的结果.
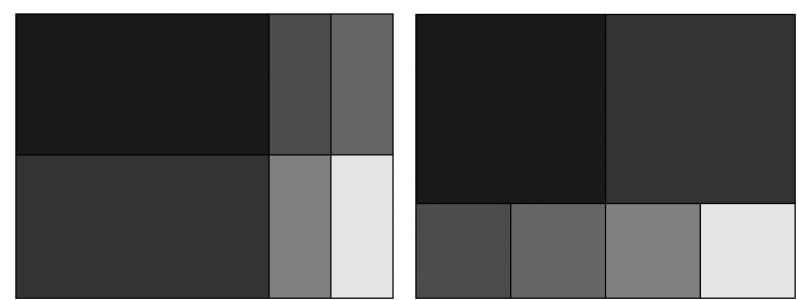

a. 沿短边布局原则
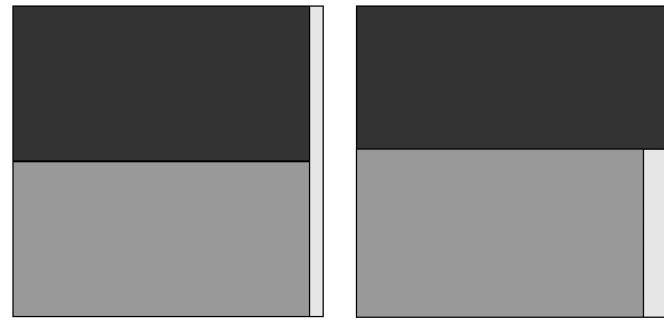

b. 贪心原则

图 $1 \mathrm{SQR}$ 的中存在的问题

\subsection{MCTS}

MCTS 是增强学习的一种算法, 可以不利用 任何先验知识，通过迭代搜索的方式对可能的结 果进行采样, 并根据采样结果做出相应的决策. 在 搜索过程中, MCTS 的搜索树是非对称增长的, 这 样即使目标问题的搜索空间很大，依旧能够在给 定条件下得到一个较好的结果 ${ }^{[21]}$

MCTS 针对不同问题有不同的变体和应用 ${ }^{[21-22]}$. 在理想条件下，假设有无限内存和时间，MCTS 可 以得到一个最优的结果. 但是在实际中, 受限于有 限的资源, 设计者往往转而追求一个次优的决策, 即在有限的迭代次数下去搜索更有价值的路径, 得到一个虽然不一定是最优但是也不错的结果, 这就涉及如何在探究和开发之间进行取舍的问题 ${ }^{[23]}$.

众所周知的 Alpha-go ${ }^{[24]}$ 就是在 MCTS 的基础 上添加价值网络以评估位置, 添加决策网络以决 定行为. MCTS 也广泛应用于一些纸牌游戏 ${ }^{[25-26]}$ 和中国象棋 ${ }^{[27]}$ 中. 与这些游戏类似, 本文要解决 的树图布局问题都要对当前的布局状态做出评估, 然后根据评估结果做决策. 由于不确定当前节点 的坐标设置会对剩余节点的布局产生什么样的影 响, 因此需要搜索可行操作. 给定一个区域以及层 次数据, 求长宽比最优的布局结果是一个 NP-hard 问题 ${ }^{[28]}$, 不可能利用常规技巧改进现有的树图布
局算法的长宽比. 因此, 可以尝试用 MCTS 对布局 的可行解空间进行迭代搜索, 并结合相应的布局 知识求解在可接受时间内长宽比较优的树图布局 结果.

\section{2 对现有图布局算法的总结与分析}

\section{1 设计空间}

Baudel 等 ${ }^{[29]}$ 总结了树图布局的设计空间, 包 括节点顺序、权值表示方式、递归原则、填充原则 和布局方向. Kong 等 ${ }^{\left[{ }^{[0]}\right.}$ 也提出树图的设计参数 (如 亮度、边缘厚度、长宽比等) 会影响人感知层次数 据的结构和数据大小. 为便于后续分析, 本文将深 人介绍节点摆放顺序、布局原则以及布局方向.

\subsection{1 节点摆放顺序}

节点摆放顺序主要有保持数据原有顺序、从大 到小和从小到大 3 种, 可根据具体的任务要求和数 据特性选取. 不强调顺序的任务可以调整节点的 原有顺序以优化布局结果的长宽比, 而强调顺序 的任务则需要保持可视化结果的顺序与数据顺序 的一致性. 常见的静态树图布局算法中, SQR 将数 据从大到小排序, APP 将数据从小到大排序, 其余 算法则不改变数据的输人顺序. 虽然这些算法的 输人顺序是固定的, 但是在布局结果上呈现的空 间顺序取决于具体的布局原则和布局方向.

\subsection{2 填充原则}

根据布局算法进行分类一般有 2 种填充原则. 第 1 种原则本文称作逐步加点, 沿着某条边一个一 个地添加节点, 直到最大长宽比变大的时候停止 加点, 每次停止加点会得到一个 row, 如图 2 所示. 第 2 种原则本文称作递归分割，按照一定的原则对 节点分组然后持续递归, 到最后只有极少数节点 时布局或在递归的同时布局. 逐步加点存在的问 题在于采用贪心原则会对后续节点的布局不友好; 递归分割存在的问题是，不根据数据的大小分布

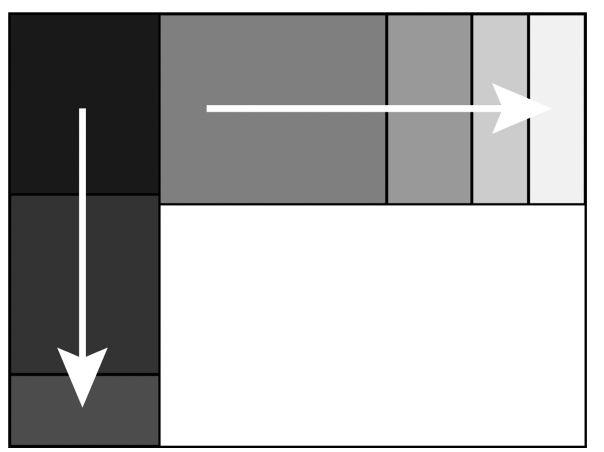

图 2 逐步加点布局原则的基本元素— row 
情况去强行地分组不太合适. 例如, Hilbert 和 Moore 算法强行把节点分为 4 组, 但是根据数据的 大小分布情况很难对数据进行 4 等分, 最后组与组 之间的差异很大. 常见的静态图布局算法中满足 逐步加点原则的是 SQR, STR 和 SPI, 满足递归分 割原则的是 Pivot, APP, Split 以及 Hilbert 和 Moore.

\subsection{3 布局方向}

布局方向一般根据人的阅读习惯和设计目标 决定. 图 3a 中, STR 的布局方向贴近人从左到右、 从上到下的阅读顺序, 但如同文本换行操作一样, 节点换行会导致相邻节点之间在空间上不连续; 图 3b 中, SQR 的布局方向相对图 3a 更混乱, 并且 会导致部分连续节点的视线位移量较大; 图 3c 中, SPI 的布局方向呈螺旋状, 解决了视线位移过大的 问题, 却对用户阅读数据不友好; 图 3d 中, Hilbert 算法根据某些规则曲线的方向进行摆放，虽不易 于阅读, 但是保留了数据的连续关系. 过分限制布 局方向会不利于优化长宽比, 并且部分算法摆放 方式比较扭曲, 对人阅读数据不友好, 不利于节点 的顺序查找.

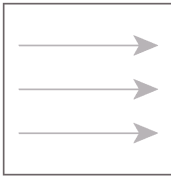

a. STR

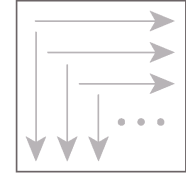

b. SQR

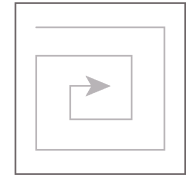

c. SPI

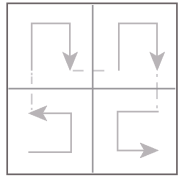

d. Hilbert
图 34 种布局方向

\section{2 满足逐步加点原则的布局算法}

常见的静态布局算法中满足逐步加点原则的 有 SQR, STR 和 SPI，这 3种算法十分有代表性. 表 1 所示为这 3 种算法满足的特性.

表 13 种树图布局算法满足的特性

\begin{tabular}{ccccc}
\hline 算法 & 平均长宽比 & 稳定性 & 一致性 & 可读性 \\
\hline $\mathrm{SPI}^{[5]}$ & 中等 & 较优 & 较优 & 中等 \\
$\mathrm{SQR}^{[6]}$ & 较优 & 较差 & 较差 & 较差 \\
$\mathrm{STR}^{[7]}$ & 中等 & 中等 & 中等 & 较优 \\
\hline
\end{tabular}

$\mathrm{SQR}$ 的平均长宽比优势最大, 但因其在布局 前对节点进行从大到小的排序操作，在布局过程 中采用贪心原则和沿短边布局原则，布局结果对 应的稳定性、数据一致性和可读性都比较差.

STR 的布局方向很符合人从左到右从上到下 的阅读顺序，具有较高可读性. 虽然它在布局前并 没有对节点进行排序，但是布局过程中逐行对节 点进行布局会导致割点处节点之间位移较大, 故
具有中等的稳定性和数据一致性, 并且依照贪心 原则确定切割点会导致最后有 “长条”出现, 布局 结果的平均长宽比往往不够好.

SPI 沿着螺旋线布局，好处是节点与节点之间 都是连续的, 不会产生断点, 故它对应的数据一致 性很好, 同时数据稳定性也较好. 但由于它沿着螺 旋线进行布局，导致用户在连续搜索节点时视线 转向次数过多, 可读性不太好. 因为 SPI 布局前没 有对节点进行排序, 所以也可能出现数据差异比 较大的节点放在一起的情况, 导致长宽比不好, 一 般布局结果具有中等的平均长宽比.

由此可见，对于常见的 4 种评价指标，这 3 种 布局算法都可以满足其中的一个或 2 个. 鉴于 STR 和 SPI 在长宽比上的劣势, 以及 SQR 对部分数据 长宽比也有优化的空间, 本文将对这 3 种算法进行 优化, 让用户可以根据不同的需求从这 3 种算法中 选择.

\section{3 本文算法}

第 2.2 节讨论过的满足逐步加点布局原则的 3 种算法(SQR, STR, SPI) 只是利用贪心原则或沿短 边布局原则得到了一些非最优的结果, 并不会进 一步判断某个节点如何摆放能够得到更优的结果. 本节首先介绍如何利用 MCTS 进行树图布局, 然 后提出加权长宽比的评估方式, 并利用 MCTS 优 化加权平均长宽比.

\section{1 针对算术平均长宽比的优化}

如图 3 所示, 3 种树图布局算法只是在输人节 点顺序和布局方向上有差异，但是在添加节点的 时候都是根据当前的方向逐个节点进行添加, 并 且直到该 row 的长宽比变差了就停止(贪心原则), 实际上, 什么时候停止加点能够得到更好的结果 都是不确定的. 根据这个布局流程，结合 MCTS, 可以建立对应的搜索树.

\subsection{1 状态节点}

每个状态节点都包含了一种布局方式，包含 的属性有当前状态节点的访问次数、胜利场数和已 固定的 row组, 还有未布局的空白区域的坐标和未 固定位置的节点组. 特例为根状态节点时, 一个节 点都没有固定，空白区域为整个画布. 如果图 2 的 布局方式作为某个状态节点的布局, 就有 2 个已固 定的 row，第 1 个 row 包含 3 个节点，方向为纵向; 第 2 个 row 包含 4 个节点, 方向为横向; 白色区域 为待布局区域(空白区域). 


\subsection{2 可行的动作}

针对不同的算法以及是否是根状态节点, 可 行的动作可能有微小的差异. 例如, 对于 SPI, 第 1 个节点的布局方向是固定的，它的子状态节点就 只有一个可选的动作, 即沿着矩形区域最上方的 边进行摆放; 其余状态节点可行的操作有把当前 节点放在上一个 row 中(如图 4 中的(3)所示), 或者 放在新建的 row 中(如图 4 中的(4)所示). 对于 STR 和 SQR, 原算法在一开始选择沿短边摆放, 但是 靠长边摆放可能得到一个更好的结果，所以在确 定第 1 个节点位置的时候改为 2 种可选的方案: (1) 沿着短边摆放 (如图 4 中的(1)所示); (2) 沿着长边 摆放 (如图 4 中的 (2)所示). 所以对应根状态节点有 2 个状态子节点. SQR 除了根状态节点以外的状态 节点的可行的动作有 3 种：(1) 加人当前 row(如图 4 中的(3)所示); (2) 新建一个 row 沿短边摆放; (3) 新建一个 row 沿长边摆放. SPI 和 STR 都只有其中 的 2 种, 具体沿长边布局还是短边布局要看其规定 的布局方向.

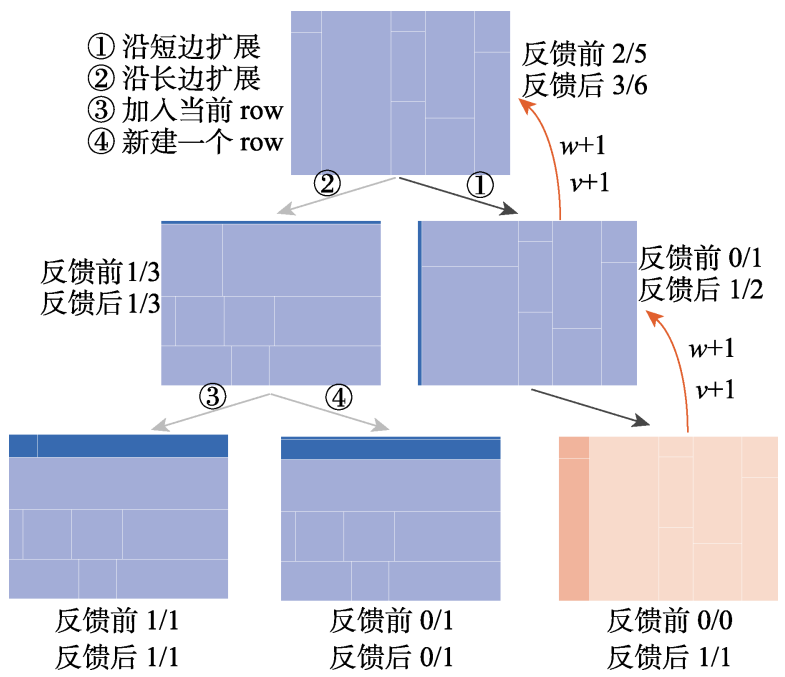

图 4 STR 对应的 TM-MCTS 搜索过程

\section{1 .3 选点公式}

在搜索过程中需要在每层中选择“价值”较大 的点以及尽量更广地探索节点, 选取的标准是置 信度上界(upper confidence bound, UCB) 公式 ${ }^{[21]}$

$$
\gamma\left(n^{\prime}\right)=\frac{w\left(n^{\prime}\right)}{v(n)}+c \sqrt{\frac{\ln (v(n))}{2 v\left(n^{\prime}\right)}}
$$

其中， $n^{\prime}$ 为状态节点 $n$ 的子状态节点； $v(n)$ 为对状 态节点 $n$ 的访问次数; $w(n)$ 表示状态节点 $n$ 对应 的胜利场次; $c$ 是一个常数, 用于控制开发新状态 节点的程度和贪心程度 (即选择胜率更高的节点的
可能性)的大小关系. 通常, 设置式(1)中的常数 $c=1^{[31]}$. 如果 $c$ 过大, 搜索宽度会相应大一些, 对 应迭代次数会比较多，在解空间很小的情况下可 以得到一个很好的结果; 但是如果解空间很大，而 $c$ 过大则需要在很多不必要的状态节点上试错, 使 得在达到了迭代阈值之后仍不能得到一个较好的 结果. 如果 $c$ 很小, 那么搜索就很接近贪心原则, 每次都选胜率很高的状态节点, 游戏很快终止, 可 能会因搜索范围过小而得到一个不好的结果. 因 此, 需要根据具体情况对 $c$ 进行调整. 当存在一些 子状态节点代人式(1)的结果一样时, 要在这些状 态节点中随机进行选点.

\subsection{4 快速模拟}

快速模拟的目的是评估当前状态下所选行为 的可能结果. 由于每个搜索到的状态节点都需要 进行这一操作，因此应保证其耗时尽量短. 本文选 择对应的原布局算法, 可以在较短时间内得到一 个比较好的结果, 降低了结果的随机性, 也在一定 程度上用到了现有布局算法的知识，从而可以有 效地进行剪枝。

\subsection{5 游戏规则}

游戏胜利定义为当前状态节点对应的平均长 宽比小于当前局面中所有状态节点对应的布局的 平均长宽比的均值. 式(1)用胜率去评估一个节点 的价值而不是直接用长宽比, 是因为这样可以降 低长宽比的影响程度, 使整个搜索过程更加平滑.

游戏结束定义为布局完最后一个节点或达到 了用户容忍极限, 即最大可迭代次数. 当解空间比 较小或式(1)中 $c$ 很小时，一般会更容易达到前一 个条件，否则会更容易达到后一个条件.

\subsection{6 本文算法步骤}

输人．画布大小 $W \times H$ ，单层数据 $D$, 最大可 忍受迭代次数 $I$, 要优化的布局算法(STR, SPI, SQR 中的一个, 用户根据具体任务进行选择).

输出. $D$ 中数据对应的布局结果.

Step1. 选点. 从根状态节点开始往下搜索, 假设当 前搜索到节点 $n$ :

Step1.1. 如果 $n$ 的可行动作都已经被执行了, 那么利用式(1)对其子状态节点进行评估, 选出最好的 子状态节点 $n^{\prime}$ (式(1)结果最大), 执行下一步; 如果 $n$ 存 在还未执行的动作, 转 Step2;

Step1.2. 如果 $n^{\prime}$ 的可行动作都被执行了, 转 Step1.1; 如果 $n^{\prime}$ 存在可行动作未被执行, 执行下一步.

Step2. 扩展. 从 $n$ 未执行的动作中随机选取一个 动作，执行该动作得到 $n^{\prime \prime}$. 
Step3. 快速模拟. 利用选取的要优化的布局算法 在空白区域对未布局的数据进行布局.

Step4. 反馈. 计算 $n^{\prime \prime}$ 在快速模拟之后对应的平均 长宽比, 如果满足游戏胜利条件, 设置 $n^{\prime \prime}$ 的胜利场次和 访问次数为 1 , 并且让其父辈节点的胜利场次和访问次 数都增加 1 ; 否则, 设置 $n^{\prime \prime}$ 的访问次数为 1 , 胜利场数 为 0 , 并把其父辈节点的访问次数都增加 1 .

Step5. 在未达到游戏终止条件之前, 循环执行 Step1 Step4. 如果达到游戏终止条件, 则返回最优布局 结果.

该算法只是针对单层层次数据进行布局的, 对于多层的数据直接在对应的单层算法上进行递 归布局即可. 图 4 所示为 STR 的 MCTS 搜索过程 STR-MC(strip treemap layout based MCTS, 其余方 式的缩写类似). 其中，蓝色的树图表示当前已经 存在的状态节点, 深蓝色表示已经固定的数据点 的位置，浅蓝色表示对空白区域进行快速模拟之 后得到的剩余数据点的布局位置. 从根状态节点 开始向下搜索，此时根状态节点的可行操作已经 执行完了，所以利用 UCB 公式选到了右边的子状 态节点. 因为右边的子状态节点还没执行任何动 作, 所以随机选择可行动作开始扩展, 得到红色的 状态节点(图 4 中黑色的箭头表示当前扩展路径), 并且该状态节点对应的长宽比优于当前搜索树的 平均水平, 故对其父辈节点的访问次数和胜利场 数都进行了加 1 操作.

\section{2 针对加权平均长宽比的优化}

在数据规模越来越大的情况下，现有显示设 备无法让所有节点都十分清晰地显示, 甚至有些 节点小到看不清. Veras 等 ${ }^{[17]}$ 提出要根据不同显示 区域的大小决定让哪些上层节点可见，让其余细 节一些的节点不可见，之后通过 “下钻”操作去看 更为细节的点. 所以，对应当前问题，细节一些的 点就是面积较小的点(大部分是底层节点), 重要的 点就是面积大一些的点(大部分是上层节点). 根据 节点大小与对应数值大小成正比这一特性，节点 $n$ 对应的加权平均长宽比定义为

$$
\alpha(n)=\frac{V(n)}{V(r)} \max \left(w_{n} / h_{n}, h_{n} / w_{n}\right)
$$

其中, $V(r)$ 表示根节点对应的目标属性值; $w_{n}$ 和 $h_{n}$ 分别为节点 $n$ 对应的宽度和高度. 由于本文加 权平均对于不同的数据可能会属于不同的范围， 不能与算术平均的数值相比较，不同数据的结果 也不能比较，因此后面只是用于在同一个数据上 进行对比分析.
利用第 3.1 节提到的 TM-MCTS 布局算法, 要 得到对应加权平均长宽比较好的布局结果, 只需 要把对应状态节点评估方式从长宽比的算术平均 改为加权平均, 对应游戏的胜利条件改为小于当 前局面所有状态节点对应的加权平均长宽比的均 值，即可得到 WTM-MCTS.

\section{4 实验及结果分析}

为了验证本文算法的有效性, 本文根据 3 种不 同分布(指数分布、高斯分布和均匀分布)及其不同 的参数设置, 以及不同的数据规模设置 $(10,20,40$, $80 ， 160 ， 320 ， 640)$ 做了 10 次乱序操作，得到 2100 个单层数据; 在这些单层数据上运行了 STR, SPI 和 SQR 原算法，对应 TM-MCTS 类型的 3 种布局 算法(STR-MC, SPI-MC 和 SQR-MC)在 3 种常见画 布比(4:3, 1: 1 和 16:9)下对应的布局结果; 还 对不同的参数设置进行对比分析. 之后, 本文从 Vernier 等 ${ }^{[4]}$ 中提供的 12 万个多层的真实数据中随 机抽取了 4000 个数据, 利用 3 种布局算法(STR, SPI 和 SQR) 和对应的 TM-MCTS, WTM-MCTS 优 化算法进行布局，并做对比分析.

\section{1 单层数据分析}

图 5 所示为 2100 个单层数据对应 6 种算法及 其不同参数设置下的布局结果，这 6 个图对应不同 节点数 $(20,40,80,160,320,640)$ 下数据的布局结 果的平均长宽比情况, 纵坐标为平均长宽比. 可以 看出, 当前数据集对应的布局的平均长宽比总是 $S T R>S P I>S Q R$ ，一般 SQR 会比前 2 种算法好一些， 原因是前 2 种算法的平均长宽比结果差异不大, 所 以相对于长宽比, STR 的优化空间比较大, SPI 次 之, SQR 有较小的优化空间. 从优化结果来看, 在 这个数据集上, TM-MCTS 算法对于 STR 的优化的 程度一般要高于 SPI, 远高于 SQR, 但是这并不能 认为对 SQR 算法的优化没有意义，如该算法对应 图 1 中的 2 个数据都能搜索到其最优的结果.

从不同的分布情况对应的结果来看, 指数分 布对应的布局结果的平均长宽比比高斯分布和均 匀分布的数据对应的结果差一些. 大部分情况下, TM-MCTS 算法对于指数分布数据的优化程度要 大一些，在指数分布时 STR 和 SPI 的平均长宽比结 果与 SQR 的差异比高斯分布和均匀分布大, 说明 数据排序会很大程度上缓解相邻数据差异过大对 布局长宽比造成的影响. 


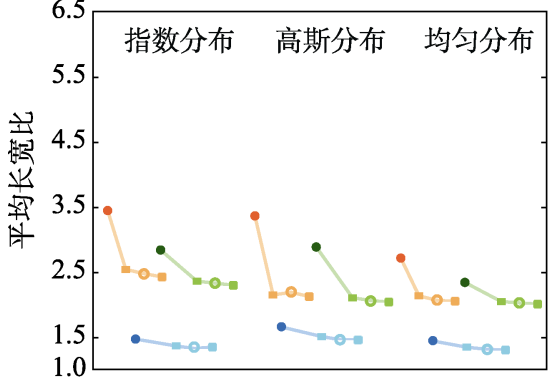

a. 节点数为 10

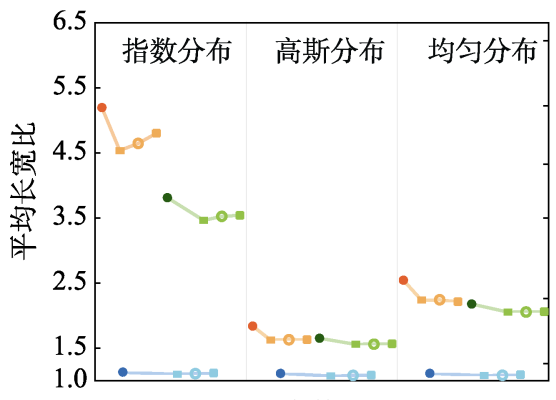

d. 节点数为 160

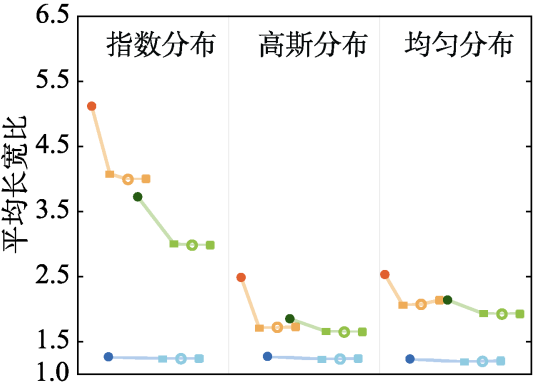

b. 节点数为 40

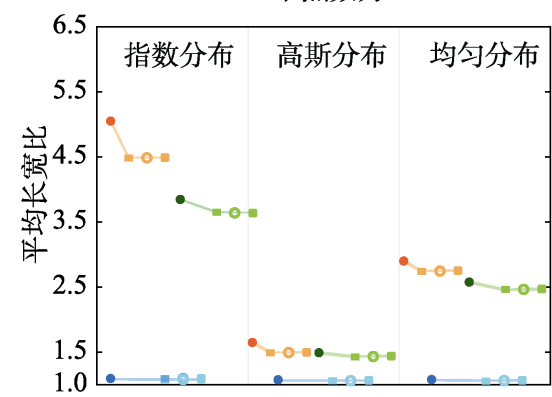

e. 节点数为 320

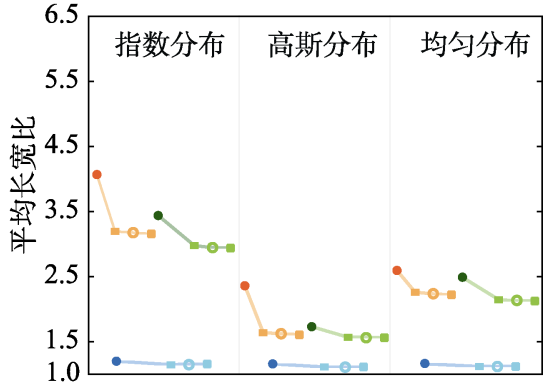

c. 节点数为 80

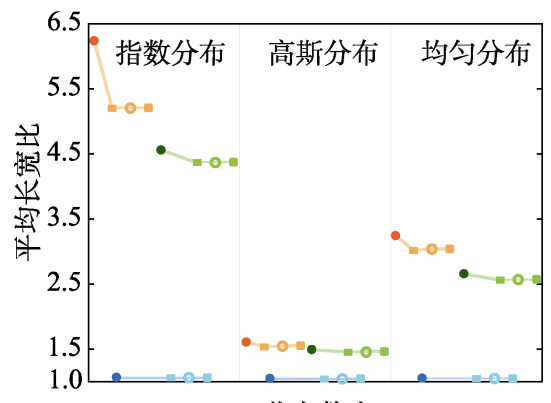

f. 节点数为 640

$\mathrm{STR}^{[7]} ; \square \mathrm{STR}-\mathrm{MC} ; \quad \mathrm{SPI}^{[6]} ; \quad \mathrm{SPI}-\mathrm{MC} ; \square \mathrm{SQR}^{[5]} ; \square \mathrm{SQR}-\mathrm{MC} ;-c=0.5 ; 0 c=1.0 ;+c=2.0$

图 5 不同布局算法在单层数据集下的结果

对于不同的参数设置 $c$, 当节点数比较少时, 解空间比较小; 探索程度越高, 越能够发现更优的 结果. 因此, 当节点数小于或等于 80 时, 大多是 $c=2.0$ 的结果比 $c=0.5$ 好. 当节点数比较多时, 解 空间比较大, 倾向于把有限的迭代次数放在尽量 好的结果上. 因此, 此时贪心的程度越高, $c$ 应该 越小. 实验结果表明, 当节点数比较多时, $c$ 越小, 得到的布局结果平均长宽比越小.

从图 5 可以看出, 算法用时主要分为选点用 时、反馈用时和布局用时, 都与具体的搜索路径和 数据规模相关; 而对应搜索路径与用户设置的最 大迭代次数和参数 $c$ 相关; 选点用时和反馈用时与 搜索树中状态节点所在的层数直接相关, 会随着 层数的升高而增大. 随着状态节点所在层数的增
大, 对应的未布局节点数在减少, 单个状态节点布 局时间会减少.

图 6 所示为 2100 个单层数据在不同的最大迭 代次数以及不同的参数 $c$ 设置下的算法平均用时 情况 (95\%置信区间). 可以看出, 随着节点数的增 加, 用时在增加; 随着最大迭代次数的增加, 用时 也在增加; 当节点数比较少时(阈值与迭代次数相 关), $c$ 越小, 对应的用时会相对较少, 但是当节点 数增加之后, 越容易达到最大迭代次数限制. 此 时, $c$ 越小反而会使搜索树相对不平衡, 选点和反 馈的用时会增加, 所以在节点数增加之后, $c$ 比较 小时用时不一定少. 从图 6 中还可以看出，限制的 最大迭代次数为 10000 时, 640 个节点的用时也不 到 $500 \mathrm{~ms}$ ，说明算法具有一定的实用性.

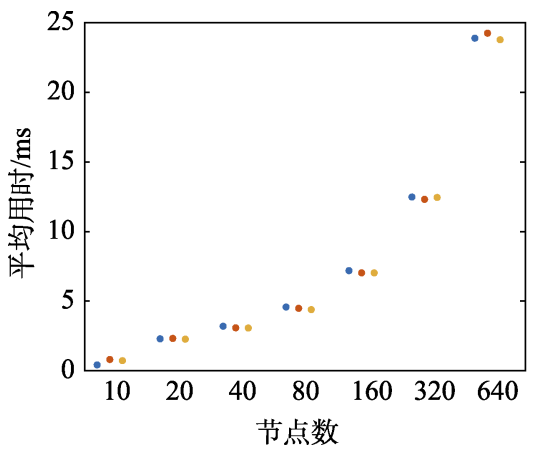

a. 最大迭代次数为 1000

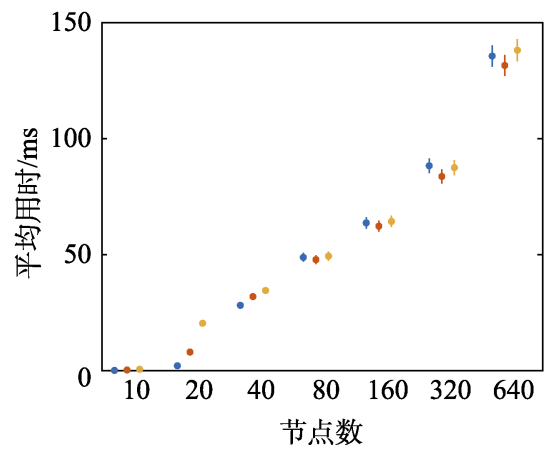

b. 最大迭代次数为 5000

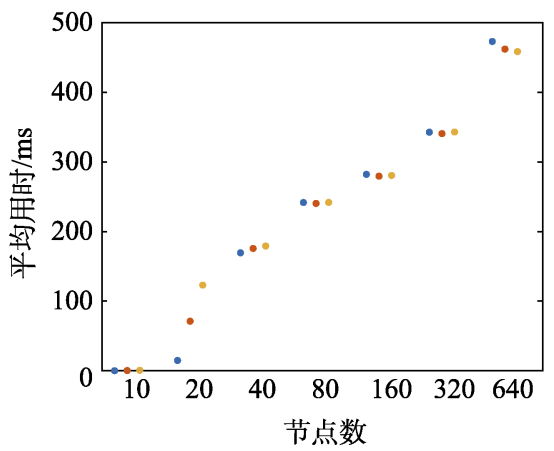

c. 最大迭代次数为 10000

图 6 不同最大迭代次数下对应的算法用时 


\section{2 多层真实数据分析}

本文通过对 Vernier 等 ${ }^{[4]}$ 的真实数据集中的 12 万 个多层数据进行随机采样, 得到了 4000 个多层的 真实数据, 并利用之前提到的 6 种算法对这些数据 的布局结果进行计算, 图 7 所示为不同算法下布局 结果对应的平均长宽比(95\%置信区间). 可以看出, 依旧是 SQR 的长宽比结果最好, 并且对应优化算 法优化的程度相对较小, 不到 1; STR 和 SPI 原算 法的长宽比结果较差, 优化空间也较大, 对应算法 确实对平均长宽比优化了很多.

对真实数据集的 gh-apollo-m.data 中的 t24.csv 进行优化, 结果如图 8 所示. 从图 8a 图 8c 中可以 看出, STR-MC 对 STR 中的长条进行了一定的优 化, 比较明显的是图中的红色区域和黄色区域，对 应 WSTR-MC 的结果也减少了很多长条, 其余地方 也有一些提升; 从图 8d 图 8f 中可以看出, SPI-MC 深蓝色区域对应的结果虽然看起来变差了, 却解

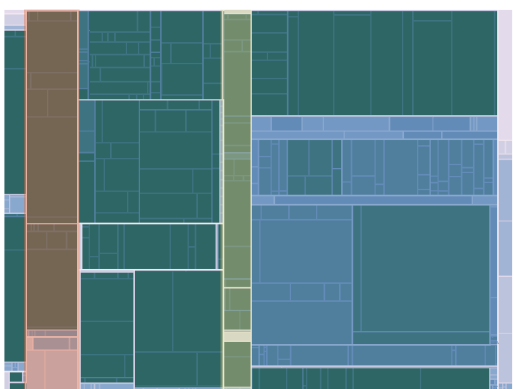

a. $\operatorname{STR}^{[7]}$

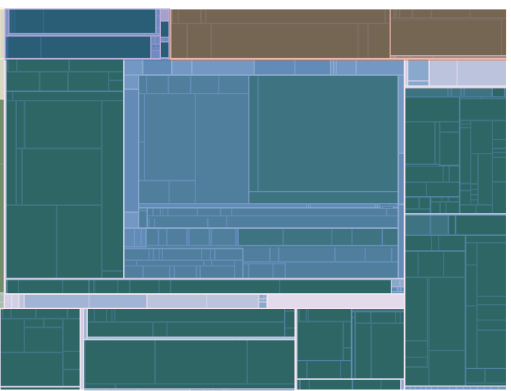

d. SPI ${ }^{[5]}$

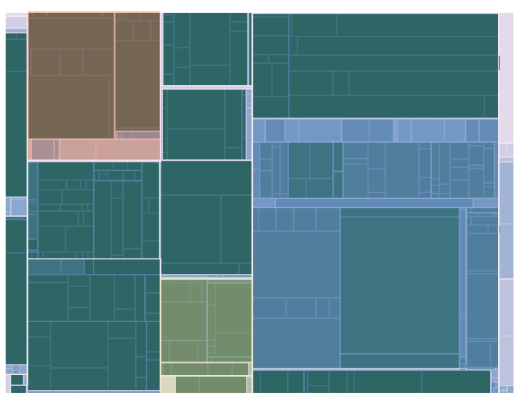

b. STR-MC

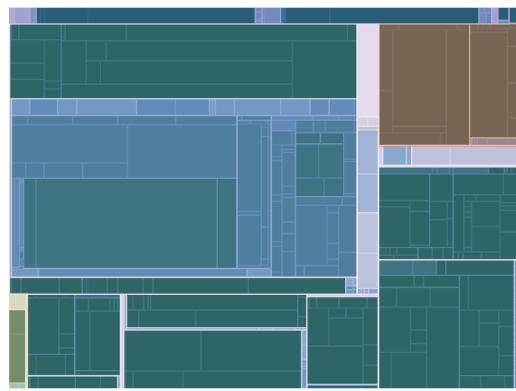

e. SPI-MC
决了黄色区域的长条问题，并且让红色区域更正 方化了, 总的平均长宽比还有提升, WSPI-MC 对 3 个区域都有提升(SQR 对应的优化程度较小，所以 未放图). 表 2 所示为这 9 种算法对应的长宽比结 果以及用时情况.

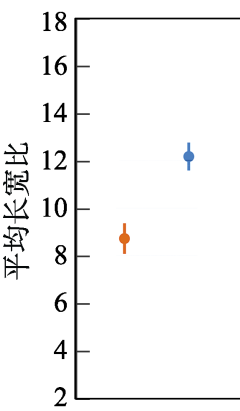

a. $\operatorname{STR}^{[7]}$

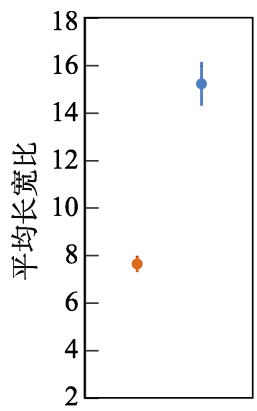

b. SPI ${ }^{[5]}$

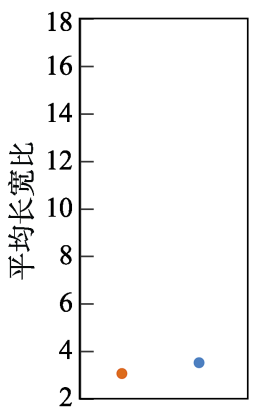

c. $\mathrm{SQR}^{[6]}$
图 73 种树图布局算法及其 TM-MCTS 优化算法在 多层数据集下的结果

图 8 STR 和 SPI 及其优化算法关于某个多层数据的结果

表 29 种算法长宽比结果和用时

\begin{tabular}{lrcr}
\hline \multicolumn{1}{c}{ 算法 } & 算术平均长宽比 & 加权平均长宽比 & 用时 $/ \mathrm{ms}$ \\
\hline $\mathrm{STR}^{[7]}$ & 22.32 & 30.07 & $\mathbf{3}$ \\
STR-MC & $\mathbf{9 . 2 9}$ & 25.08 & 512 \\
WSTR-MC & 10.84 & $\mathbf{2 2 . 4 2}$ & 324 \\
SPI $^{[5]}$ & 13.15 & 38.30 & $\mathbf{4}$ \\
SPI-MC & $\mathbf{1 0 . 6 4}$ & 33.51 & 127 \\
WSPI-MC & 14.21 & $\mathbf{2 3 . 5 2}$ & 64 \\
SQR $^{[6]}$ & 5.75 & 18.78 & $\mathbf{6}$ \\
SQR-MC $^{[6.92}$ & $\mathbf{5 . 1 0}$ & 21.98 & 1346 \\
WSQR-MC & 5.86 & $\mathbf{1 8 . 3 3}$ & 621 \\
\hline
\end{tabular}

注. 粗体代表该算法在相应指标下结果较好.
从表 2 中可以看出, 10 层以上的数据在配置不 高的笔记本电脑(i5-8265U CPU, 8 GB 内存)上也能 在较短的时间内完成布局, 并得到一个长宽比更 优的结果; STR 的算术平均长宽比优化程度最大, SPI 次之, SQR 较小; SPI 的加权平均长宽比优化程 度最大, STR 次之, SQR 较小.

\section{3 对布局算法选取的建议}

综上所述, 不是对所有的数据一定要选择 TM-MCTS 进行优化, 也不是对所有的数据都一定要 
把 $c$ 设置得很小或是很大, 需要根据不同的情况做 出决定. 本文针对不同的情况提出以下建议.

（1）如果不要求数据稳定性以及其他特性, 只 强调布局结果的长宽比, 并且利用 SQR 计算得出 的长宽比已经很小了 (如长宽比接近 $1.5^{[30]}$ ), 说明 再用 TM-MCTS 也很难对布局有比较大的优化并 且会耗费时间，此时可以直接使用 SQR 进行布局. 但是如果 SQR 的结果看起来不是很好, 可以用 SQR-MC 尝试优化长宽比.

（2）如果要求数据稳定性和数据一致性, 但是 不要求布局结果具有良好的可读性, 那么可以利 用 SPI 布局; 如果 SPI 布局结果的平均长宽比较大, 就使用 SPI-MC 进行优化.

（3）如果要求要保证布局结果的稳定性和数 据一致性，并且要易于用户阅读，那么可以选择 STR, 一般对应的 STR 的长宽比结果都比较差, 可 以使用 STR-MC 对其进行优化.

（4）如果点数很少，可以直接使用 TM-MCTS 进行布局，并且可以把 $c$ 值设置得稍微大一些; 如 果点数比较多, 要把参数 $c$ 值设置得稍微小一些, 或者考虑增加最大迭代次数阈值，用更多的时间 换取更好的结果.

\section{5 结 语}

本文先根据树图布局的原则对常用的静态树 图布局算法进行分类. 由于满足逐步加点原则的 3 种树图布局算法 (SQR, STR 和 SPI) 在常见的 4 种指 标下各有优势, 但是 STR 和 SPI 的长宽比往往不 好, SQR 的长宽比针对部分数据有提升的必要; 所 以本文结合 MCTS 对这 3 种算法的长宽比进行了 优化, 并针对 “数据规模比较大的时候可以先显示 重要的节点”这一设计思想, 提出加权平均长宽比 这一指标, 并对式(1)进行修改, 得到对加权平均 长宽比进行优化的 WTM-MCTS. 之后, 利用对应 算法在不同的单层人造数据以及多层真实数据上 进行了对比分析，根据其分析结果对用户选择布 局算法提出了指导意见.

本文算法还存在一些缺陷：一是现有算法对 于多层数据是在单层数据布局的基础上进行递归 布局，没有考虑层与层之间的相互影响，如某些布 局算法对当前层的数据可以得到长宽比较好的布 局结果，但是会使下一层的节点难以得到长宽比 较好的布局; 二是当前算法没有在式(1)的基础上 设计对应的剪枝算法, 在点数较多时不仅用时长,
而且优化结果也不够好, 还需要进一步研究; 三是 现在还只是在数值层面上考虑了某个指标对于布 局的影响，没有设计严谨的用户实验验证优化算 法是否能使用户在特定任务中提升效率和准确率.

从 WTM-MCTS 算法可以看出, TM-MCTS 系 列算法可以有各种各样的衍生算法, 本文仅尝试 了改变 TM-MCTS 中状态节点的评估方式，从算术 平均改为了加权平均, 就可以得到符合不同要求 的布局结果. 当前只是针对长宽比优化, 将来可以 针对树图的其他指标修改搜索树的结构和评估方 式得到符合其他标准的算法，或者也可以利用 MCTS 算法对符合递归分割布局原则的布局算法 求取更好的切割点用以递归布局。

\section{参考文献(References):}

[1] Balzer M, Deussen O, Lewerentz C. Voronoi treemaps for the visualization of software metrics[C] //Proceedings of the ACM Symposium on Software Visualization. New York: Association for Computing Machinery, 2005: 165-172

[2] Görtler J, Schulz C, Weiskopf D, et al. Bubble treemaps for uncertainty visualization[J]. IEEE Transactions on Visualization and Computer Graphics, 2018, 24(1): 719-728

[3] Onak K, Sidiropoulos A. Circular partitions with applications to visualization and embeddings[C] //Proceedings of the 24th Annual Symposium on Computational Geometry. New York: ACM Press, 2008: 28-37

[4] Vernier E, Sondag M, Comba J, et al. Quantitative comparison of time-dependent treemaps[J]. Computer Graphics Forum, 2020, 39(3): 393-404

[5] Tu Y, Shen H W. Visualizing changes of hierarchical data using treemaps[J]. IEEE Transactions on Visualization and Computer Graphics, 2007, 13(6): 1286-1293

[6] Bruls M, Huizing K, van Wijk J J. Squarified treemaps[C] // Proceedings of the Data Visualization 2000. Heidelberg: Springer, 2000: 33-42

[7] Bederson B B, Shneiderman B, Wattenberg M. Ordered and quantum treemaps: making effective use of $2 \mathrm{D}$ space to display hierarchies[J]. ACM Transactions on Graphics, 2002, 21(4): 833-854

[8] Shneiderman B. Tree visualization with tree-maps: 2-D spacefilling approach[J]. ACM Transactions on Graphics, 1992, 11(1): 92-99

[9] Shneiderman B, Wattenberg M. Ordered treemap layouts[C] // Proceedings of the IEEE Symposium on Information Visualization. Los Alamitos: IEEE Computer Society Press, 2001: 73-78

[10] Wood J, Dykes J. Spatially ordered treemaps[J]. IEEE Transactions on Visualization and Computer Graphics, 2008, 14(6): 1348-1355

[11] Engdahl B. Ordered and unordered treemap algorithms and their applications on handheld devices[D]. Stockholm: Royal Institute of Technology, 2005

[12] Tak S, Cockburn A. Enhanced spatial stability with Hilbert and 
Moore treemaps[J]. IEEE Transaction on Visualization and Computer Graphics, 2013, 19(1): 141-148

[13] Zhu Xingyu, Du Jinglin. A squarified treemap layout algorithm based on mean dichotomy drive blockwise sorting[J]. Journal of Computer-Aided Design \& Computer Graphics, 2015, 27(8): 1451-1459(in Chinese)

(朱兴宇，杜景林. 均值二分法分块排序的正方化树图布局算 法[J]. 计算机辅助设计与图形学学报, 2015, 27(8): 1451-1459)

[14] Vliegen R, van Wijk J J, van der Linden E J. Visualizing business data with generalized treemaps[J]. IEEE Transactions on Visualization and Computer Graphics, 2006, 12(5): 789-796

[15] Zhang Chi, Xiang Xiaoxue, Zhang Pengzhou. Research and optimization of visual layout algorithm based on squarified treemap[J]. Computer Engineering and Applications, 2017, 53(9): 208-212(in Chinese)

(张弛, 向小雪, 张鹏洲. 正方化树图布局算法研究与优化 [J]. 计算机工程与应用, 2017, 53(9): 208-212)

[16] Blanch R, Lecolinet E. Browsing zoomable treemaps: structure-aware multi-scale navigation techniques[J]. IEEE Transactions on Visualization and Computer Graphics, 2007, 13(6): 1248-1253

[17] Veras R, Collins C. Optimizing hierarchical visualizations with the minimum description length principle[J]. IEEE Transactions on Visualization and Computer Graphics, 2017, 23(1): 631-640

[18] Nagamochi H, Abe Y. An approximation algorithm for dissecting a rectangle into rectangles with specified areas[J]. Discrete Applied Mathematics, 2007, 155(4): 523-537

[19] Lu L F, Fan S L, Huang M L, et al. Golden rectangle treemap[J]. Journal of Physics: Conference Series, 2016, 787(1): Article No.012007

[20] Liu Xu. Squarified treemap layout algorithm based on depth-first search[J]. Computer Systems \& Applications, 2017, 26(5): 105-112(in Chinese) (刘旭. 基于深度优先搜索的正方化树图布局算法[J]. 计算 机系统应用, 2017, 26(5): 105-112)
[21] Browne C B, Powley E, Whitehouse D, et al. A survey of Monte Carlo tree search methods[J]. IEEE Transactions on Computational Intelligence and AI in Games, 2012, 4(1): 1-43

[22] Maes F, St-Pierre D, Ernst D. Monte Carlo search algorithm discovery for single-player games[J]. IEEE Transactions on Computational Intelligence and AI in Games, 2013, 5(3): 201-203

[23] Auer P, Cesa-Bianchi N, Fischer P. Finite-time analysis of the multiarmed bandit problem[J]. Machine Learning, 2002, 47(2): 235-256

[24] Silver D, Huang A, Maddison C J, et al. Mastering the game of Go with deep neural networks and tree search[J]. Nature, 2016, 529(7587): 484-489

[25] Rubin J, Watson I. Computer poker: a review[J]. Artificial Intelligence, 2011, 175(5/6): 958-987

[26] Whitehouse D, Powley E J, Cowling P I. Determinization and information set Monte Carlo tree search for the card game Dou Di Zhu[C] //Proceedings of the IEEE Conference on Computational Intelligence and Games (CIG'11). Los Alamitos: IEEE Computer Society Press, 2011: 87-94

[27] Nijssen J A M, Winands M H M. Enhancements for multiplayer Monte-Carlo tree search[C] //Proceedings of International Conference on Computers and Games. Heidelberg: Springer, 2010: 238-249

[28] Beaumont O, Boudet V, Rastello F, et al. Matrix multiplication on heterogeneous platforms[J]. IEEE Transactions on Parallel and Distributed Systems, 2001, 12(10): 1033-1051

[29] Baudel T, Broeksema B. Capturing the design space of sequential space-filling layouts[J]. IEEE Transactions on Visualization and Computer Graphics, 2012, 18(12): 2593-2602

[30] Kong N, Heer J, Agrawala M. Perceptual guidelines for creating rectangular treemaps[J]. IEEE Transactions on Visualization and Computer Graphics, 2010, 16(6): 990-998

[31] Kocsis L, Szepesvári C, Willemson J. Improved Monte-Carlo search[OL]. [2020-09-26]. https://www.researchgate.net/publication/228341626_Improved_monte-carlo_search 